\title{
Mesial temporal lobe epilepsy: an old and yet not entirely unfold story
}

\author{
Epilepsia do lobo temporal mesial: uma história antiga e não inteiramente contada \\ Luciano de Paola ${ }^{1,2}$, Vera Cristina Terra ${ }^{1,2}$
}

\footnotetext{
Universidade Federal do Paraná,

Serviço de Neurologia, Programa de Epilepsia, Curitiba PR, Brazil;

2 Hospital Nossa Senhora das Graças, Centro de Diagnóstico e Tratamento de Epilepsia (EPICENTRO), Curitiba PR, Brazil.
}

\section{Correspondence:}

Luciano de Paola; Av. Sete de Setembro, 4848 / conj

1105; 80240-000 Curitiba PR, Brasil;

luciano.depaola@gmail.com

Conflict of interest:

There is no conflict of interest to declare.

Received 15 January 2015 Accepted 20 January 2015

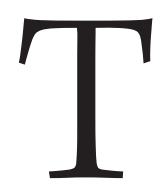

he World Health Organization (WHO) website, on its Mental Health Programmes section, suggests an interesting call of duty: "Epilepsy: Treat it. Defeat it". It highlights the fact that over 50 million people have epilepsy worldwide, $80 \%$ of whom living in developing countries, where 3 out of 4 persons with epilepsy do not receive appropriate care for their seizure disorder ${ }^{1}$. The reader is then directed to the International League Against Epilepsy page, where an opening banner reads: "Epilepsy is still a puzzle"2. The combination of these thought provoking statements brings to mind Sun Tzu's Art of War ${ }^{3}$ often cited quotation: "know your enemy". Even with mesial temporal lobe epilepsy (MTLE) standing as the most systematically studied form of the disease and hippocampal sclerosis (HS) representing the most common pathology underlying its drug-resistant cases ${ }^{4}$, this remains a somewhat "unknown" and challenging opponent. Thus, every piece of information counts on trying to understand the intricacies on the clinical presentation of this subset of patients. In that sense, the paper by Coan and coworkers $^{5}$, featured in this issue of Arquivos de Neuropsiquiatria, describing similar proportion of relapse-remitting seizure pattern in MTLE patients with and without HS is a welcome one and it certainly raises a couple of considerations. Over the past decade or so the epilepsy community has shown a trend towards more pragmatic definitions. Epilepsy (now a disease, instead of a condition) is defined by the occurrence of at least two unprovoked seizures over $24 \mathrm{hr}$ apart or even one unprovoked (or reflex) seizure and a probability of further seizures of at least $60 \%{ }^{6}$. Drug-resistant epilepsy is established as failure of adequate trials of 2 tolerated, appropriately chosen and used antiepileptic drug schedules to achieve sustained seizure freedom ${ }^{7}$. In epilepsy management, these definitions represent shortcuts to potential surgical indications. Some 14 years ago, Wiebe's pivotal paper on the New England Journal of Medicine ${ }^{8}$ showed clearly better outcomes on surgical, as opposed to medically treated, patients on a randomized study involving refractory temporal lobe epilepsy cases (70\% of whom with MRI proven HS). Regardless the unquestionable quality of these data, a tempting approach emerged with an embedded (and equivocal) idea that MTLE with HS cases translate, almost by default, into surgical cases. That also includes an excuse to rather "minimalistic" work-ups (sometimes the mere history of epilepsy and an MRI imaging of HS) leading to surgical indications. That could eventually be the case, should all HS were created alike. But they are not, as elegantly demonstrated by ILAE's Commission on Diagnostic Methods and its international consensus of HS in TLE ${ }^{9}$. The committee reported both interobserver and intraobserver agreement on 3 types of HS, referring to different patterns of neuronal cell damage. HS ILAE type I is likely to concur with a better outcome, as compared to types 2 and 3. This data has been recently validated on a large cohort of MTLE with HS cases ${ }^{10}$. In this survival analysis, HS ILAE type I patients (severe neuronal cell loss and gliosis in CA1 and CA4 regions) showed better long term seizure outcome, as well as, differences on clinical history when matched with HS ILAE types 2 and 3. Inevitably, the hypothesis of subsets within the subset comes to mind when thinking MTLE with (or without) HS. In their paper, Coan and coworkers ${ }^{5}$ arbitrarily defined relapse-remitting pattern of seizures as a period equal or longer than 2 years without any type of epileptic seizures in 172 consecutive patients with clear cut diagnosis of MTLE (122 with HS and 50 without). Roughly a third in each group presented with a relapse-remitting pattern demonstrating that: (a) HS patients may experience 
fairly long seizure remission, even being medically intractable and (b) this very feature - i.e, a relapse-remitting seizure pattern - is of little help as a clinical tool separating lesional vs nonlesional MTLE. More so, $11 \%$ of their MTLE with HS patients were seizure free for fairly long periods (some up to 9 years, as per Coan's description). These prolonged remissions (given the occurrence of relapses) do not prevent surgical indications, but on selected cases, patients maybe prone to decline the surgical option and choose to risk an occasional relapse. In other words, not all MTLE with HS patients are necessarily predestinated to the surgical arena. The authors recognize that data in children are scarce. Although MTLE is frequently reported in pediatric population, almost all published data focus in comparing kids and adults in respect to clinical semiology and surgical outcome. One study that evaluated seizure outcome in surgical and non-surgical children with MTLE comes from Sales et $\mathrm{al}^{11}$. Here 31 children $(<12$ y/o) were followed for 3 years, with MTLE (with HS, NL or tumoral etiology). Eight patients had significant seizure improvement with pharmacotherapy: 3 patients with MTLEHS had seizure control and one a seizure reduction (one seizure each 4 months) and 4 patients with MTLE-NL had seizure control. These findings are close to those observed in Coan's description. Authors also observed an inverse relationship between age at seizure onset and seizure frequency, suggesting that patients with earlier seizure onset had a worst outcome. However, some questions remain unanswered. Since MTLE, especially when related to HS, is a disease that need circuitry reorganization are we talking about the same thing? Children with MTLE usually present with simple clinical semiology and more diffuse interictal and ictal EEG patterns ${ }^{12}$. Are these secondary to brain maturation or children with MTLE are "small adults" that will continue to seize during life, with possible periods of relative control? If on one hand, one cannot avoid a little frustration at the prospect that what seemed to be our most consolidated epileptic syndrome (MTLE with HS) remains very much unknown in many aspects, on the other findings such as Coan's data calls for double attention and even more educated decision making trees on MTLE cases. Plus, one should keep in mind distinctive patterns of MTLE and HS may impact not only on seizure prognosis but perhaps on diverse cognitive and even behavioral curses of the disease. As "little strokes fell great oaks"13, Coan's welcome paper may have added a little piece to the epilepsy puzzle.

\section{References}

1. World Health Organization - WHO. WHO programme on reducing the epilepsy treatment gap. Geneva: World Health Organizationh; 2015 [cited 2015 jan 13]. Available from: http://www.who.int/mental_health/neurology/epilepsy/en/

2. International League Against Epilepsy. ILAE topic-oriented commissions. WestHartford:International LeagueAgainstEpilepsy;2015 [cited 2015jan 13].Available from: http://www.ilae.org/Visitors/Commissions/Topic.cfm

3. Sun Tzu. The art of war: classic edition. El Paso: El Paso Norte; 2009.

4. Malmgren K, Thom M. Hippocampal sclerosis: origins and imaging. Epilepsia; 2012;53(suppl 4):19-33. http://dx.doi.org/10.1111/j.1528-1167.2012.03610.x

5. Coan AC, Campos BM, Bergo FPG et al. Patterns of seizure control in patients with mesial temporal lobe epilepsy with and without hippocampus sclerosis. Arq Neuropsiquiatr. 2015;73(2):79-82.

6. Fischer RS, Acevedo C, Arzimanoglou A, Bogacz A, Cross JH, Elger CE et al. ILAE oficial report: a practical clinical definition of epilepsy. Epilepsia. 2014;55(4):475-82. http://dx.doi.org/10.1111/epi.12550

7. Kwan P, Arzimanoglou A, Berg AT, Brodie MJ, Hauser WA, Mathern $G$ et al. Definition of drug resistant epilepsy: consensus proposal by the ad hoc Task Force of the ILAE Commission on Therapeutic Strategies. Epilepsia. 2010;51(6):1069-77. http://dx.doi.org/10.1111/j.1528-1167.2009.02397.x
8. Wiebe S, Blume WT, Girvin JP, Eliasziw M. A randomized, controlled trial of surgery for temporal-lobe epilepsy. N Engl J Med. 2001;345(5):311-8. http://dx.doi.org/10.1056/NEJM200108023450501

9. Blümcke I, Thom M, Aronica E, Armstrong DD, Bartolomei F, Bernasconi A et al. International consensus classification of hippocampal sclerosis in temporal lobe epilepsy: a Task Force report from the ILAE Commission on Diagnostic Methods. Epilepsia. 2013;54(7):1315-29. http://dx.doi.org/10.1111/epi.12220

10. Na M, Ge H, Shi C, Shen H, Wang Y, Pu S et al. Long-term seizure outcome for international consensus classification of hippocampal sclerosis: a survival analysis. Seizure. 2014 (in press). http://dx.doi.org/10.1016/j.seizure.2014.10.006

11. Sales LV, Velasco TR, Funayama S, Ribeiro LT, Andrade-Valença LP, Neder L etal. Relative frequency, clinical, neuroimaging, and postsurgical features of pediatrictemporallobeepilepsy.BrazJ Med Biol Res.2006;39(10):136572. http://dx.doi.org/10.1590/S0100-879X2006001000013

12. Cersósimo R, Flesler S, Bartuluchi M, Soprano AM, Pomata H, Caraballo R. Mesialtemporallobeepilepsywithhippocampalsclerosis:studyof42children. Seizure. 2011;20(2):131-7. http://dx.doi.org/10.1016/j.seizure.2010.11.002.

13. FranklinB.Quotation.Oqueéisso?Nãoéverbete.In:BowmanJL.Provocative and contemplative quotations: with author comments and observations. Bloomington: Authorhouse; 2013. www.authorhouse.com, 2013. 